\title{
BMJ Open Experience of living with type 1 diabetes in a low-income country: a qualitative study from Liberia
}

\author{
Alma J Adler (10 , ${ }^{1,2}$ Celina Trujillo, ${ }^{1,3}$ Leah Schwartz, ${ }^{4}$ Laura Drown, ${ }^{1,2}$ \\ Jacquelin Pierre, ${ }^{5}$ Christopher Noble, ${ }^{2}$ Theophilus Allison, ${ }^{5}$ Rebecca Cook, ${ }^{5}$ \\ Cyrus Randolph, ${ }^{5}$ Gene Bukhman ${ }^{1,2,3}$
}

To cite: Adler AJ, Trujillo C, Schwartz L, et al. Experience of living with type 1 diabetes in a low-income country: a qualitative study from Liberia. BMJ Open 2021;11:e049738. doi:10.1136/ bmjopen-2021-049738

- Prepublication history and additional supplemental material for this paper are available online. To view these files, please visit the journal online (http://dx.doi.org/10.1136/ bmjopen-2021-049738).

$\mathrm{CR}$ and $\mathrm{GB}$ are joint senior authors.

Received 03 February 2021 Accepted 29 September 2021

\section{Check for updates}

(c) Author(s) (or their employer(s)) 2021. Re-use permitted under CC BY-NC. No commercial re-use. See rights and permissions. Published by BMJ.

${ }^{1}$ Division of Global Health Equity, Department of Medicine, Brigham and Women's Hospital, Boston, Massachusetts, USA

${ }^{2}$ Program in Global

Noncommunicable Disease and Social Change, Harvard Medical School Department of Global Health and Social Medicine, Boston, Massachusetts, USA ${ }^{3}$ Partners in Health, Boston, Massachusetts, USA

${ }^{4}$ Harvard Medical School,

Boston, Massachusetts, USA

${ }^{5}$ Partners in Health Liberia, Harper, Liberia

Correspondence to

Dr Alma J Adler

aadler2@bwh.harvard.edu

\section{ABSTRACT}

Introduction While epidemiological data for type 1 diabetes (T1D) in low/middle-income countries, and particularly low-income countries (LICS) including Liberia is lacking, prevalence in LICs is thought to be increasing. T1D care in LICs is often impacted by challenges in diagnosis and management. These challenges, including misdiagnosis and access to insulin, can affect T1D outcomes and frequency of severe complications. Despite the severe nature of T1D and growing burden in subSaharan Africa, little is currently known about the impact of T1D on patients and caregivers in the region.

Methods We conducted a qualitative study consisting of interviews with patients with T1D, caregivers, providers, civil society members and a policy-maker in Liberia to better understand the psychosocial and economic impact of living with T1D, knowledge of T1D and selfmanagement, and barriers and facilitators for accessing T1D care.

Results This study found T1D to have a major psychosocial and economic impact on patients and caregivers, who reported stigma, diabetes distress and food insecurity. Patients, caregivers and providers possessed the knowledge necessary to effectively manage T1D but insufficient community awareness leads to delayed diagnosis, often in an emergency department. Most patients reported receiving free services and materials, though the cost of transportation to clinic visits and recommended foods is a barrier to disease management. Many providers noted the lack of national T1D-specific guidelines and registries. Policy-makers reported a lack of prioritisation of and resources for T1D. These barriers, combined with scarcity and expense of appropriate foods, pose severe barriers for selfmanagement of T1D.

Conclusion T1D was found to have a significant impact on patients and caregivers, and informants identified several key individual and systems-level barriers to effective T1D care in Liberia. Addressing these concerns is vital for designing sustainable and effective programmes for treating patients living with T1D.

\section{INTRODUCTION}

If you meet today a person that just got diabetes, and the person is feeling bad, what would you tell them? I will tell them sorry.

\section{STRENGTHS AND LIMITATIONS OF THIS STUDY}

$\Rightarrow$ First qualitative study of type one diabetes in a lowincome country.

$\Rightarrow$ One of the first studies in sub-Saharan Africa to comprehensively interview people living with type one diabetes, their caregivers, healthcare providers, pharmacists, civil society and policy-makers.

$\Rightarrow$ This study includes patients from five different health facilities in three regions of Liberia representing diverse models of healthcare and funding.

-A conversation between a clinician and person living with type 1 diabetes in rural Liberia

Type 1 diabetes (T1D) is a severe chronic autoimmune disease in which the pancreas produces little or no insulin. It typically presents in young people and necessitates artificial insulin replacement therapy. ${ }^{1}$ Epidemiological data for T1D in low and middleincome countries (LMICs) is lacking, ${ }^{2}$ but prevalence is estimated at $0.012 \%$ in sub-Saharan Africa (SSA) and thought to be increasing. Average life expectancy is estimated at fiveyears after diagnosis and patients with T1D without access to proper care generally do not survive one year. ${ }^{3}$ There are limited data on life expectancy following initial diagnosis of T1D in high-income countries but this is estimated at over 40 years. ${ }^{4-6}$

People living with T1D (PLWT1D) require daily insulin injections to maintain appropriate blood glucose (BG) levels. A recent study suggested that in LMICs, the price of insulin is a major barrier, and is available in about $50 \%$ of public facilities and less than $40 \%$ of private facilities. ${ }^{2}{ }^{7}$ While the median buyer price for one vial of insulin was US $\$ 4.31$ globally from 1996 to 2013 , in LMICs it was higher, at US $\$ 6.90 .{ }^{8}$ The main cause of mortality for PLWT1D globally is lack of access to insulin, reflecting shortcomings in availability and affordability. ${ }^{3}$ 
T1D care in LMICs is affected by numerous challenges in diagnosis and management. Late diagnosis and misdiagnosis remain common, influenced by lack of awareness and familiarity with T1D and access to diagnostic resources; there is often a greater focus on endemic infectious diseases at the expense of chronic ones. Inadequate management impacts patient outcomes and frequency of severe complications, including challenges in adjusting insulin doses given limited home glucose monitoring and food insecurity, high levels of infectious disease and complications such as diabetic ketoacidosis (DKA) and hypoglycaemia. ${ }^{9}$

In an assessment of paediatric diabetes care in 64 countries, Liberia was one of six countries receiving the lowest Life for a Child (LFAC) index score, a measure which captures availability of essential components of care, including insulin, BG monitoring, diabetes education and complications screening. ${ }^{10}$ Access to care for PLWT1D in Liberia is very limited and only provided in a few centres by organisations including Partners in Health (PIH) in facilities in Pleebo and Harper (Maryland County) and the International Diabetes Federation's LFAC programme at facilities in Monrovia (Montserrado County), and Ganta (Nimba County).

Despite the severity and increasing burden of T1D in SSA, there is little research on the impact of T1D on patients and caregivers, especially in low-income countries (LIC). This study is the first to examine the impact of T1D on patients, caregivers and health systems across Liberia, and the first qualitative study in this area in a LIC in SSA. In this study, we examine the psychosocial and economic impact of living with T1D, knowledge of T1D and self-management, and barriers and facilitators for accessing T1D care.

\section{METHODS}

\section{Framework}

To identify PLWT1D and healthcare provider experience relating to diabetes management, we used a framework using theories from implementation research ${ }^{11}$ and behaviour change. ${ }^{12}$ The framework encapsulates barriers to diabetes management and facilitates exploration of mediating pathways and moderators. ${ }^{13}$

\section{Data collection}

Interviews were conducted in October to December 2019 by a trained nurse practitioner (CT) and physician assistant (CR) in English or Liberian English. Both researchers were currently practising in Harper. All participants gave signed informed consent prior to being interviewed. Standardised semistructured interview scripts (online supplemental file 1) were used and sessions were audio recorded. Study informants included one from the $\mathrm{MOH}$, two from diabetes civil society organisations (LFAC and Diabetes and Endocrine Society of Liberia (DESOL)), and informants recruited from five health facilities in Monrovia and two rural regions of Liberia known to
Table 1 Informant characteristics

\begin{tabular}{|c|c|c|}
\hline Characteristic & $\begin{array}{l}\text { Number } \\
(n=28)\end{array}$ & Percentage \\
\hline \multicolumn{3}{|l|}{ Stakeholder type } \\
\hline Patient & 10 & 35.7 \\
\hline Caregiver & 5 & 17.9 \\
\hline Provider & 10 & 35.7 \\
\hline Endocrinologist & 1 & 10.0 \\
\hline Paediatrician & 2 & 20.0 \\
\hline Internist & 2 & 20.0 \\
\hline Physician assistant & 1 & 10.0 \\
\hline Nurse & 3 & 30.0 \\
\hline Pharmacist & 1 & 10.0 \\
\hline Civil society member & 2 & 7.1 \\
\hline Policy-maker & 1 & 3.6 \\
\hline \multicolumn{3}{|l|}{ Site } \\
\hline Ministry of Health, Monrovia & 1 & 3.6 \\
\hline JFK Medical Center & 4 & 14.3 \\
\hline JDJ Hospital & 4 & 14.8 \\
\hline $\begin{array}{l}\text { Ganta United Methodist } \\
\text { Hospital }\end{array}$ & 7 & 25.0 \\
\hline J.J. Dossen Hospital & 9 & 32.1 \\
\hline Pleebo Health Center & 3 & 10.7 \\
\hline \multicolumn{3}{|l|}{ Sex } \\
\hline Female & 17 & 60.7 \\
\hline \multirow[t]{2}{*}{ Male } & 11 & 39.3 \\
\hline & Mean (SD) & Range \\
\hline Patient age (years) & $18.9(6.8)$ & $10-30$ \\
\hline
\end{tabular}

provide care for PLWT1D (table 1): (1) JJ Dossen (JJD) Hospital in Maryland County, (2) Pleebo Health Center in Maryland County, (3) John F Kennedy Medical Center (JFK) in Monrovia, (4) James Davis Junior (JDJ) Memorial Hospital in Monrovia, and (5) Ganta United Methodist Hospital in Ganta. T1D clinics in Ganta and JDJ are funded through LFAC and provide free insulin and materials to all PLWT1D 26 years and younger. PIH provides assistance to clinics in Maryland Country, including provision of free insulin and materials for PLWT1D. Interview topics varied by informant (table 2). Interviews were conducted until thematic saturation was achieved. Patients to be interviewed were selected based on age, availability and willingness to participate. We interviewed five patients with a secondary school education, two with less than a secondary school education, and three with a college education. We interviewed three patients between 10 and 18, and seven over the age of 18 . We interviewed families of younger patients.

\section{Data analysis}

Interviews were transcribed and, when necessary, translated directly from Liberian English to English during 
Table 2 Interview question topics

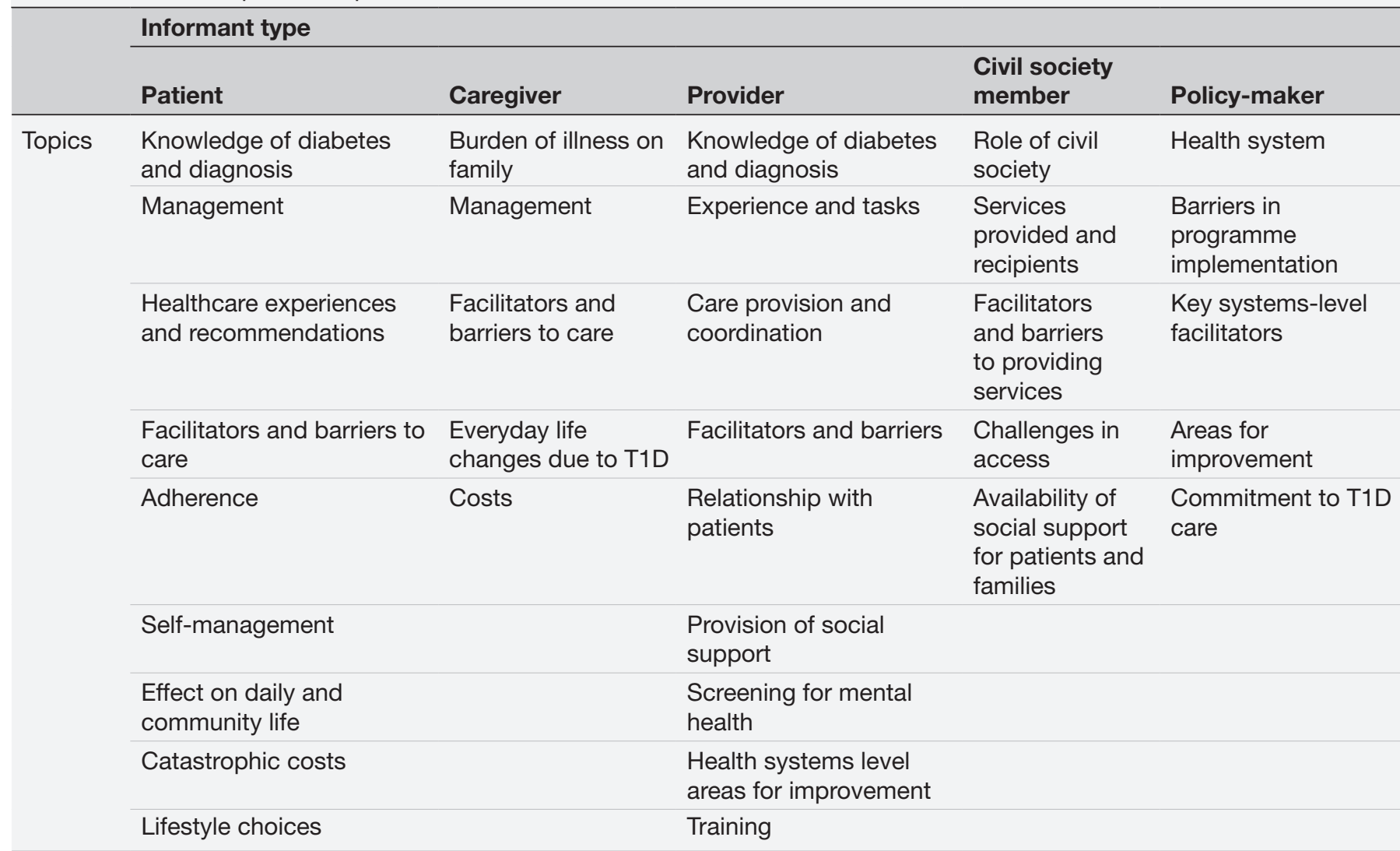

T1D, type 1 diabetes.

transcription. All coding was conducted in Dedoose. ${ }^{14}$ The subsequent analysis was comprised of two iterative steps. We began with a-priori thematic analysis and, after initial coding based on these themes, expanded our coding structure to encompass additional themes that emerged through the first step of analysis. Three of the four researchers involved in analysis have no relation to the community where study participants were recruited. The fourth resided in Maryland County for three months and participated in medical care for some participants. We held a virtual meeting between individuals involved in analysis to discuss major themes and issues that arose from the analysis. To ensure quality of results, all researchers independently coded a representative sample of interviews and met to ensure consistency across the coding structure. The revised coding structure was used to recode the initial interview sample and all subsequent interviews. Results were reported using Standards for Reporting Qualitative Research.

\section{Patient and public involvement}

The aim of this study was to understand the experience of PLWT1D, particularly their priorities, experiences and preferences. Research questions and measures were designed with the help of an author $(\mathrm{CN})$ who lives with T1D. This author was involved in protocol development and interview guide design.
PLWT1D in Liberia were actively recruited and interviewed in this study. As part of informed consent, they were informed of the time required to participate.

\section{RESULTS}

\section{Psychosocial/economic impact of T1D}

Patient\#7: ... my life changed because since I was diagnosed in 2014, I stopped going out with my friends...not clubbing. or nothing, normally we used to go play football, sometimes we [hung out]. But ... its always 'My man, that stuff I can't eat, I don't do that.' or 'My man let's do JBC [communal contributions to food]' and everybody is putting money together... Someone else, 'Oh my man you're selfish.' But I really didn't want to tell him why the reason I was doing it. So I would say I was close with them but all of a sudden I became selfish and other things. And it hurt me a lot.

Informants identified alienation from peers at school as an important contributor to psychosocial stress. They reported feeling worried that people felt sorry for them and feelings alienated from inability to consume the same foods as their peers. Indeed, some reported not eating in front of others. Others reported worrying about injecting insulin in front of their peers. Overall, PLWT1D shared a significant desire to hide their T1D diagnosis from peers. PLWT1D reported skipping insulin doses 
when participating in sports or physical labour due to a fear of hypoglycaemia, especially in the context of food insecurity.

Diabetes distress refers to the accumulation of everyday stresses among individuals with diabetes over time as they struggle with managing their T1D. ${ }^{15}$ This concept was reflected in responses from PLWT1D and providers, especially surrounding daily insulin injections and fingersticks. One provider discussed how children will run from their caregivers when they try to inject insulin, while another caregiver commented on the extreme distress her daughter experienced around fingersticks:

Caregiver\#2: Sometimes she feels so bad. You know, when she's at play and I call her to come and do the test, she says, 'hey man, I'm tired with this sickness, I just want to die now. Every day, all my fingers hurting, I'm tired.'

Many caregivers discussed feeling overwhelmed by the difficulty of caring for their loved ones with T1D. They noted the burden of financial stress, amount of time spent actively helping loved ones manage T1D, and fear of leaving them alone.

One aspect of T1D's broader economic impact in Liberia is its effect on the ability of PLWT1D and their caregivers to attend school and work. While some younger PLWT1D and their caregivers discussed attending school, one PLWT1D reported leaving school due to T1D. PLWT1D and providers explained that patients may also be unable to work, especially when feeling poorly due to complications of T1D.

\section{Knowledge and self-management}

PLWT1D and caregivers described the signs and symptoms leading to their T1D diagnosis including the ' 3 Ps' of hyperglycaemia (polydipsia (excessive thirst), polyphagia (constant hunger), and polyuria ('going pee-pee fast-fast')), glycosuria (ants attracted to their urine), gastrointestinal issues (vomiting and abdominal pain), weight loss (their body 'reducing' or 'getting dry' or 'being to dry dry'), weakness and fatigue, malaise (feeling 'not myself', 'feeling different', or 'getting/being sick'), mood swings, a sense of dread, altered mental status and frequent fungal infections. Only providers and civil society mentioned symptoms suggesting potentially lifethreatening situations, including confusion and loss of consciousness. Most PLWT1D reported checking their own BG levels at home, typically two to three times per day. Several patients mentioned recording their readings in logbooks for providers to use for adjusting insulin dosages. Many identified readings constituting high or low BG and explained how they would respond to them. Others reported not checking BG levels at home and relying on readings from clinic visits. Importantly, PLWT1D correctly identified signs of hypoglycaemia and its causes, including skipping meals and taking too much insulin. The foods informants mentioned as treatment for hypoglycaemia included sugar, rice and juice. Despite knowing they were at risk for hypoglycaemia and how treat it, only two informants reported keeping something in their room overnight or carrying something with them at all times for treating potential hypoglycaemic episodes.

PLWT1D and caregivers identified foods including bulgur wheat and green plantain as diabetic friendly or discussed diet as a way of controlling diabetes. Some discussed modifying meal sizes rather than avoiding foods with a high carbohydrate content. Two caregivers reported preparing food for their children to take to school to help them avoid eating the 'wrong' foods.

\section{Interviewer Have you ever missed a time you were supposed to take your insulin?}

Patient\#9 (Ganta): Yes. Sometimes if I feel normal in my body, I feel reluctant to take my insulin and check my sugar.

All PLWT1D correctly identified the two types of insulin and reported being on a standard regimen of two daily injections, with adjustments for high or low BG. Almost half reported missing insulin doses occasionally for reasons including forgetfulness, being in a rush to go to school/work, running out, insufficient funds, or feeling reluctant to take insulin or check their BG that day. Although providers and civil society members reported patients reducing insulin dose to make their insulin last longer, no PLWT1D and only one caregiver whose sister aged out of the LFAC programme reported decreasing insulin doses due to a lack of money.

\section{Misdiagnosis or late diagnosis}

Providers reported that patients tend to receive the initial diagnosis of T1D at the emergency department (ED) in a state of crisis (often due to DKA) rather than an outpatient clinic, likely due to a lack of awareness and knowledge of T1D in the community and possibly among frontline providers. Most PLWT1D and caregivers reported no knowledge of T1D prior to diagnosis and were not previously acquainted with anyone with T1D; those who had a family member or close contact living with T1D were more likely to recognise early symptoms of the disease. While providers were aware of T1D symptoms, patients were sometimes misdiagnosed with malaria as it is endemic to the region and better known to providers. Some patients with new onset T1D were treated for other conditions and providers only considered T1D after their status did not improve. Providers and caregivers discussed how achieving a correct diagnosis required persistence and advocacy by families.

Providers reported T1D is generally diagnosed clinically with basic labs including point-of-care glucose testing and urinary ketones due to unavailability of traditional laboratory tests including C-peptide and antibodies. Clinicians have developed effective ability to diagnose and manage T1D with available resources. Providers reported using haemoglobin A1c, random BG, urinalysis, chemistries and glucose tolerance tests for diagnosis. Several providers mentioned the use of urinary ketones in the absence of serum ketones and arterial blood gases. Providers use 
patient's age, basic labs and urine ketones to differentiate between T1D and T2D.

\section{Barriers and facilitators}

Provider\#9: It's challenging for younger children to adhere to treatment. Because their food insecurity is high, there is peer pressure, the patient goes to school, their family is not there and they are used to ... eating along with their friends, they are a peer group.

\section{Individual-level barriers and facilitators}

Providers identified numerous barriers faced by PLWT1D, including low numeracy, literacy and health literacy, poor adherence to diet recommendations, high costs and limited access to insulin. For some PLWT1D and caregivers, low literacy and numeracy affect comprehension of diabetes education and self-management activities, and patients with low literacy typically require ongoing counselling to improve adherence.

Unlike other regions of the world, diabetes education in Liberia does not primarily focus on the importance of regular exercise as most Liberians have a lifestyle that requires them to be more active based on work and walking for basic needs. Education is more focused on diet. All informants mentioned the challenges of adhering to diet recommendations, related to peer pressure, cost of food, and food availability. One provider suggested that patients eat less frequently than they report. A common concern was inability to afford foods including vegetables and bulgur wheat, which are more expensive than rice. One interpreter poignantly summarised this sentiment:

Interpreter: People telling her that the condition she has, the diabetes, is a rich people sickness. That you have to buy all kinds of vegetables, all kinds of stuff, and they tell you not to eat rice. But for her, she's from a poor family. Her family cannot afford all those vegetables, those extra things to balance her diet. So sometimes she keeps herself hungry because she doesn't want to eat rice for her sugar to raise up. Sometimes it's a worry for her. Sometimes she just has to manage and eat rice and her sugar goes up. All those things are a worry for her. It's a problem.

Food insecurity also impacts patients' BG levels and insulin use. They may skip insulin doses or take insulin without food, increasing their risk for severe hypoglycaemia. Families often face the difficult choice of paying for food instead of insulin, supplies and transport to the clinic. A physician in Monrovia described dealing with this difficult situation:

Provider\#6: Once the relatives of a patient know they have to spend this amount of money for a medication to control this, they make a choice very quickly. They look at daily bread for everybody and choose that first. Whenever they get extra money to buy insulin they do that, and it's very painful. That's the major challenge. And now, they also have to check their glucose levels, to buy the machine and the strips, that's costly too. Everything around care, especially at home, becomes difficult. I see the stress from patients and relatives,... you try to negotiate the amount of times they can do testing, and where you can get this medication, that's when we're calling LFAC or sending them to places where they can get free insulin and what have you. And that's a great difficulty, especially if they can't even afford to pay their way to where the free insulin is, or to get food for their family members on a daily basis. So that's the major challenge.

One provider in Maryland County discussed the unique approach of providing food assistance to ease the burden of food insecurity among their patients. Specifically, the clinic has started providing rice, vegetables, and fruits.

PWT1D and their families incur significant expenses related to management of the disease. For those not receiving subsidised care, inability to afford insulin and other supplies was a major challenge identified by patients, caregivers, providers and civil society members. Though PLWT1D identified consistent availability of insulin at clinics as a facilitator, in some settings it was not always accessible due to cost. Informants noted this potentially led to lower adherence, particularly in reducing or skipping doses, and distress for patients.

For patients not receiving subsidised care, purchasing insulin and related supplies including test strips, syringes, and ice for storage is costly. Informants explained that patients typically spend US\$20 to US\$30 per vial of insulin, up to US $\$ 60$ a month for two vials, an unaffordable amount for many patients, most of whom make between US $\$ 100$ and US $\$ 200$ per month. PLWT1D worry about insufficient finances for insulin and having to skip or reduce doses. Providers reiterated that patients did not always take recommended doses of insulin due to cost.

Provider\#9: If you have patients buying their own insulin, especially with the economy we are faced with in this country it's going to be difficult telling most patients there is nothing we can do but just to allow you to die.

For PLWT1D receiving free insulin and supplies, burdensome expenses still exist. Across facilities, informants identified transportation costs contributing to significant out-of-pocket expenses and missed appointments. At Ganta and JJD, patients come from as far as Ivory Coast and Monrovia to receive subsidised care.

In addition to these routine expenses, PLWT1D admitted at some hospitals without free care for complications like DKA may face steep bills on discharge as some hospitals have admission fees, daily bed fees and medications or lab charges. In other hospitals, most services are free, but there are expenses including lost income of patient and caregiver attending them in the hospital, expenses for items not provided or available at the hospital. Providers explained that these bills are often unaffordable for patients, who must raise funds or seek help from the hospital.

Provider\#8: Sometimes they will need fluid, in crisis sometimes, they spend up to fifteen, twenty, thirty thousand [Liberian dollars]. Sometimes to even raise that money after 
they have been discharged...we have to go beg the hospital, please help them. right now, the hospital is in crisis, so the hospital doesn't even have enough supply before money.

PLWT1D receiving free services and medications at facilities subsidising care reported it enabled them to manage their T1D. PLWT1D living far from the clinic were given large supplies of insulin, sometimes lasting multiple months between clinic visits, and reported that this gave them more flexibility and less pressure when it was difficult to attend clinic appointments. Some patients acknowledged that this helped them to stay healthy.

PLWT1D receiving care at LFAC-supported facilities face the challenge of ageing out of the programme at age 27. A provider explained that after ageing out, patients must purchase their own insulin and supplies. The pathway for transition of patients out of this programme is unclear. It is unclear if patients receiving care at these facilities are aware of the forthcoming transition, as this was not mentioned by any patients.

Insulin starts to degrade at room temperature and spoils if stored above $32^{\circ} \mathrm{C}$. Due to the warm climate in Liberia, insulin storage was identified as a substantial barrier to effective T1D management. Only three informants reported being able to store their insulin in a refrigerator and reported problems with electricity availability. Informants reported storing their insulin in banana trees by cutting small openings into the tree trunk and in coolers, with or without ice.

\section{Systems-level barriers and facilitators}

A policy-maker explained that few resources are directed towards T1D care in Liberia, reflected in a lack of trained staff, supply chain issues and lack of funds at the facility level.

Policymaker: Often people will die very young without being diagnosed. As a result, people don't see those cases and when you want to do advocacy they will tell you we don't see those cases. But we know those cases occur, but before they are diagnosed they pass off and they are buried and then no one knows them... The figures are not there, the people don't see it, so it's difficult to raise awareness, and because we are not responding, people are not aware, so it's a kind of circle... Then the other side to it, because there has not been a lot of focus on the area the resources for care have been scarce. So how do you go out to even say you are creating awareness when the people come and you don't have anything to give them?

This informant expressed that in addition to the longstanding prioritisation of and resource allocation towards communicable diseases such as malaria, tuberculosis and HIV, there remains a lack of data demonstrating T1D burden in Liberia, making it difficult to advocate for greater allocation of resources for T1D. Much of this could be due to the lack of T1D registries, and the fact that T1D and T2D are generally grouped together when data are collected.
Providers and policy-makers recognise the need for national guidelines-while there are guidelines for the management of all diabetes emergencies developed by DESOL, there are no national T1D guidelines. Some providers reported using LFAC guidelines, while others reported using WHO guidelines, or T2D guidelines. Some providers at PIH-supported sites also reported using protocols developed by PIH. Notably, providers from the same facilities reported using different guidelines and many said that the only visible guidelines were for T2D.

Nearly all PLWT1D and caregivers reported receiving T1D education at the clinic. Providers explained that in addition to orienting patients on diagnosis, they typically provide education and counselling on a regular basis. During clinic visits, PLWT1D received education on selfmanagement activities including checking BG levels and use of insulin, insulin storage, diet, potential complications, and recognising symptoms of hyperglycaemia and hypoglycaemia. Supportive companions are also involved in education since they play a key role in supporting disease management, particularly for younger patients. One provider expressed that most patients respond well to education and use the knowledge in adhering to selfmanagement activities.

Many providers and the policy-maker reported underdiagnosis was a significant problem related to low community awareness of the disease and lack of adequate provider knowledge to recognise and correctly diagnose the disease.

Provider\#7: There could be a lot of people out there with the condition that they don't know about. And even providers that are not trained will not recognize it. But I think there needs to be more awareness and more publicity regarding this.

Informants explained that, as previously discussed, this lack of awareness can lead to later diagnosis in an acute state or even lack of diagnosis prior to T1D-related death.

\section{DISCUSSION}

This study found T1D to have major psychosocial and economic impacts on PLWT1D and their families. Patients and caregivers reported high levels of stigma, diabetes distress and food insecurity. Many patients reported challenges with correctly storing insulin. Reassuringly, both PLWT1D and caregivers possessed the knowledge necessary to effectively manage T1D, though a lack of community knowledge and awareness leads to delayed diagnosis and many PLWT1D presenting in the ED. Many patients reported receiving free services and materials. However, while PIH and LFAC-assisted sites provide these services and materials for free, other sites across the country do not, and LFAC sites only provide materials through the age of 26. Even patients who receive free services and materials, the cost of transportation to clinic visits and recommended foods are barriers to effective disease management. Many providers noted 
the lack of T1D specific guidelines and registries. Policymakers reported a lack of prioritisation of and resources for T1D. These barriers, combined with scarcity and expense of appropriate foods, pose severe barriers for self-management of T1D. Addressing these concerns is vital for designing sustainable and effective programmes for treating PLWT1D.

\section{Comparison with other studies}

There is a notable lack of qualitative research on T1D in LMICs, particularly in rural areas. A recent review of publications yielded only five qualitative studies of T1D in SSA, all from middle-income countries, including two conducted in South Africa, one in Accra, Ghana and one in Ghana including patients with T1D and T2D. ${ }^{16-19}$ The remaining study, conducted in Zambia, included patients, caregivers and health practitioners, and investigated stress and quality of life in PLWT1D. ${ }^{20}$ These authors similarly found high levels of stress, disclosure avoidance and caregiver burnout. ${ }^{20}$ The study conducted in Accra identified barriers faced by families of PLWT1D reflecting those identified by informants in this study, including misdiagnosis, the impact of school on selfmanagement, and the financial burden of the disease. To our knowledge, however, this study is the largest, most comprehensive qualitative study of T1D in SSA, and the first from a LIC.

As noted, very little has been written on T1D in LICs, but studies of T2D have highlighted depression, stigma and diabetes distress or burnout. ${ }^{20-24}$ Despite the implications for T1D management, very little has been written on techniques for insulin storage in hot countries with low levels of refrigeration. Ogle et al did a thorough analysis of 12 traditional techniques of refrigeration from seven countries, including goat skins and clay pots, with varying levels of effectiveness, although none were included that were reported in this study. ${ }^{25}$ A review of the literature did not find any studies reporting on banana trees for insulin storage. Other authors have found food insecurity to be a factor in management in all types of diabetes, although less has been written solely in the context of T1D. ${ }^{192627}$

Our study identified challenges with diabetes management related to numeracy and literacy levels in the population. There are few studies in LMICs that have addressed this issue. A recent study in Ahmedabad Gujarat, India showed that an estimated $70 \%$ of rural PLWT1D were insufficiently educated to manage their own conditions. ${ }^{28}$ On the other hand, two studies from one hospital in Tanzania found that caregiver and patient educational levels were not significantly associated with glycaemic control or frequency of complications. ${ }^{29} 30$

This study had several limitations. Despite interviewer's best efforts, it was often challenging to find quiet places to conduct interviews, which caused several interruptions. Patients and caregivers with higher education levels tended to be more likely to speak, and some PLWT1D were interviewed by people they may have seen as providers and therefore been less likely to speak negatively of the programme. Finally, most of the patients and caregivers interviewed received care at PIH or LFAC centres, and therefore received subsided services and are not representative of the broader population.

\section{Future implications}

Efforts are underway to expand T1D in SSA and elsewhere both through focused T1D initiatives and integrated PEN-Plus strategies. ${ }^{31-35}$ These strategies aim for at least intermediate levels of glycaemic control that will require diabetes self-management education. Ultimately, greater allocation of resources toward T1D care and services in Liberia is needed to address systems-level and individual-level barriers. ${ }^{2}$

This study identified areas of both health system improvement and social protection that are needed to improve the quality of T1D care in LICs. Providers interviewed highlighted the importance of standardised record keeping and guidelines, while patients emphasised the need to address food insecurity, stigma and insulin storage.

Advocacy efforts for additional T1D resources must be supported by data highlighting the burden of this disease. Thus, including regional or national systems of electronic medical records (EMR) or other methods of monitoring patient care and outcomes are needed. EMR are not only needed to assess the magnitude of the problem, but also as part of quality improvement efforts, and have been shown to lead to better patient care and clinical outcomes, and subsequently significantly reduce morbidity and mortality associated with T1D complications. ${ }^{36}$

T1D guidelines will also raise awareness of T1D among governments, providers, public health practitioners and advocates, caregivers and patients and point towards useful resources. ${ }^{37}$ Additionally, they can assist providers, PLWT1D and caregivers in managing diabetes in a standardised, evidence-based way to ensure safe care in a timely manner, taking into account the local context and resource availability. ${ }^{37-40}$ Morbidity and mortality will be improved through the use of T1D-specific guidelines, as they will enable clinicians to effectively prioritise objectives as they relate to diabetes management and care, provide guidance on how to support PLWT1D and their caregivers, and enable standard screening for T1D complications. ${ }^{38-41}$

The findings from this study have important implications for ongoing T1D programme design. More research is needed to better quantify the impact of these issues on PLWT1D in more diverse settings. However, it is already clear from this report that interventions such as food packages or cash transfers may be necessary for highquality T1D care in high-poverty settings. Additionally, 
educational initiatives and opportunities for patients with T1D and families to build and become engaged in T1D communities, such as patient advocacy and support groups may be necessary to address societal stigma for this disease. Finally, additional research on local solutions and providing improved insulin storage containers and the development of heat-stable insulins may be a priority. Indeed, while comprehensive recommendations to revise T1D programmes as described above are beyond the scope of this paper, our findings serve as a strong starting point for using a behaviour change framework to implement such changes, which is the goal of future work. ${ }^{11}$

\section{CONCLUSION}

This study fills an important gap in the existing literature as the first study conducted in a LIC in SSA to examine the impact of T1D on patients, caregivers and health systems. We found T1D has a major psychosocial impact on PLWT1D and caregivers. While patients and caregivers possessed adequate knowledge to effectively manage T1D, informants identified important individual-level and systems-level barriers to T1D care in Liberia. These findings demonstrate the need for additional research to quantify the impact of these issues and initiatives to address barriers to care in high-poverty settings such as Liberia.

Acknowledgements We are grateful for the participation of all informants of this study, to the Ministry of Health of Liberia for their support, and to LFAC for welcoming us to their facilities and introducing us to their patients.

Contributors Design of project and tools: AJA, CT, CN, LS, CR and GB. Data acquisition and management: CT, JP, TA, RC and CR. Data analysis: AJA, CT, LD and LS. Manuscript drafting: AJA, CT, LD, LS and GB. Guarantor: AJA. All authors contributed to final manuscript.

Funding This work was supported by grant 2018PG-T1D051 from the Leona M. and Harry B. Helmsley Charitable Trust.

Competing interests None declared.

Patient consent for publication Not applicable.

Ethics approval This study obtained ethics from both the Harvard Longwood Campus review board and University of Liberia Institutional Review Board \#FWA00004982. It conformed to all principles embodied in the Declaration of Helsinki.

Provenance and peer review Not commissioned; externally peer reviewed.

Data availability statement Data are available upon reasonable request. Deidentified quotes of informants taken from qualitative interviews are available upon request from the corresponding author, AJA (ORCID: https://orcid.org/00000002-6700-3279). The codebook utilized for analysis is also available.

Supplemental material This content has been supplied by the author(s). It has not been vetted by BMJ Publishing Group Limited (BMJ) and may not have been peer-reviewed. Any opinions or recommendations discussed are solely those of the author(s) and are not endorsed by BMJ. BMJ disclaims all liability and responsibility arising from any reliance placed on the content. Where the content includes any translated material, BMJ does not warrant the accuracy and reliability of the translations (including but not limited to local regulations, clinical guidelines, terminology, drug names and drug dosages), and is not responsible for any error and/or omissions arising from translation and adaptation or otherwise.

Open access This is an open access article distributed in accordance with the Creative Commons Attribution Non Commercial (CC BY-NC 4.0) license, which permits others to distribute, remix, adapt, build upon this work non-commercially, and license their derivative works on different terms, provided the original work is properly cited, appropriate credit is given, any changes made indicated, and the use is non-commercial. See: http://creativecommons.org/licenses/by-nc/4.0/.

\section{ORCID iD}

Alma J Adler http://orcid.org/0000-0002-6700-3279

\section{REFERENCES}

1 World Health Organization. Definition and diagnosis of diabetes mellitus and intermediate hyperglycemia: report of a WHO/IDF consultation, 2006.

2 Atun R, Davies JI, Gale EAM, et al. Diabetes in sub-Saharan Africa: from clinical care to health policy. Lancet Diabetes Endocrinol 2017;5:622-67.

3 Beran D, Yudkin JS. Diabetes care in sub-Saharan Africa. Lancet 2006;368:1689-95.

4 Huo L, Harding JL, Peeters A, et al. Life expectancy of type 1 diabetic patients during 1997-2010: a national Australian registrybased cohort study. Diabetologia 2016;59:1177-85.

5 Livingstone SJ, Levin D, Looker HC, et al. Estimated life expectancy in a Scottish cohort with type 1 diabetes, 2008-2010. JAMA 2015;313:37.

6 Petrie D, Lung TWC, Rawshani A, et al. Recent trends in life expectancy for people with type 1 diabetes in Sweden. Diabetologia 2016;59:1167-76.

7 Beran D, Hirsch IB, Yudkin JS. Why are we failing to address the issue of access to insulin? a national and global perspective. Diabetes Care 2018;41:1125-31.

8 Ewen M, Beran D, Joosse H-J. Insulin prices profile. Amsterdam: Health Action International, 2016.

9 Bahendeka SK. Diabetes in sub-Saharan Africa: let us not forget type 1. Lancet Diabetes Endocrinol 2017;5:575-7.

10 Ogle GD, Middlehurst AC, Silink M. The IDF life for a child program index of diabetes care for children and youth. Pediatr Diabetes 2016;17:374-84.

11 Michie S, Johnston M, Abraham C, et al. Making psychological theory useful for implementing evidence based practice: a consensus approach. Qual Saf Health Care 2005;14:26-33.

12 Michie S, Atkins L, West R. The behaviour change wheel: a guide to designing intervention. London: Silverback Publishing, 2014.

13 Davies P, Walker AE, Grimshaw JM. A systematic review of the use of theory in the design of guideline dissemination and implementation strategies and interpretation of the results of rigorous evaluations. Implement Sci 2010;5:14.

14 Dedoose Version 8.3.35, web application for managing, analyzing, and presenting qualitative and mixed metho research data [program] 2020.

15 Diabetes Distress Assessment \& Resource Center, 2017. Available: https://diabetesdistress.org/ [Accessed 25 Sep 2020].

16 Brown O, Fouché P, Coetzee M. Bouncing forward: families living with a type I diabetic child. South African Family Practice 2010;52:536-41.

17 Jonker D, Deacon E, van Rensburg E, et al. Illness perception of adolescents with well-controlled type 1 diabetes mellitus. Health Psychol Open 2018;5:205510291879996.

18 Kratzer J. Structural barriers to coping with type 1 diabetes mellitus in Ghana: experiences of diabetic youth and their families. Ghana Med J 2012;46:39-45.

19 de-Graft Aikins A. Healer Shopping in Africa: new evidence from rural-urban qualitative study of Ghanaian diabetes experiences. BMJ 2005;331:737.

20 Hapunda G, Abubakar A, van de Vijver F, et al. Living with type 1 diabetes is challenging for Zambian adolescents: qualitative data on stress, coping with stress and quality of care and life. BMC Endocr Disord 2015;15:20.

21 Edah JO, Goar SG, Odoh G, et al. Undiagnosed depression among adults with diabetes mellitus in Jos. Niger J Clin Pract 2020;23:1431-6.

$22 \mathrm{~K}$ Wolde A, G Wondim M. Diabetic distress among diabetic patients in the referral hospital of Amhara regional state, Ethiopia. Int $Q$ Community Health Educ 2020;40:105-14.

23 Mendenhall E, Musau A, Bosire E, et al. What drives distress? Rethinking the roles of emotion and diagnosis among people with diabetes in Nairobi, Kenya. Anthropol Med 2020;27:252-67.

24 Mogre V, Johnson NA, Tzelepis F, et al. Barriers to diabetic selfcare: a qualitative study of patients' and healthcare providers' perspectives. J Clin Nurs 2019;28:2296-308.

25 Ogle GD, Abdullah M, Mason D, et al. Insulin storage in hot climates without refrigeration: temperature reduction efficacy of clay POTS and other techniques. Diabet Med 2016;33:1544-53. 
26 Cheng S, Kamano J, Kirui NK, et al. Prevalence of food insecurity in patients with diabetes in Western Kenya. Diabet Med 2013;30:e215-22.

27 Ogunrinu T, Gamboa-Maldonado T, Ngewa RN, et al. A qualitative study of health education experiences and self-management practices among patients with type 2 diabetes at Malamulo Adventist hospital in Thyolo district, Malawi. Malawi Med J 2017;29:118.

28 Pacaud D, Lemay J-F, Richmond E, et al. Contribution of sweet to improve paediatric diabetes care in developing countries. Pediatr Diabetes 2016;17 Suppl 23:46-52.

29 Noorani M, Ramaiya K, Manji K. Glycaemic control in type 1 diabetes mellitus among children and adolescents in a resource limited setting in Dar es Salaam - Tanzania. BMC Endocr Disord 2016;16:29.

30 Majaliwa ES, Munubhi E, Ramaiya K, et al. Survey on acute and chronic complications in children and adolescents with type 1 diabetes at Muhimbili national hospital in Dar ES Salaam, Tanzania. Diabetes Care 2007;30:2187-92.

31 World Health Organization Regional Office for Africa. WHO pen and integrated outpatient care for severe, chronic NCDS at first referral hospitals in the African region (PEN-Plus): report on regional consultation, 2019.

32 Tapela NM, Bukhman G, Ngoga G, et al. Treatment of noncommunicable disease in rural resource-constrained settings: a comprehensive, integrated, nurse-led care model at public facilities in Rwanda. Lancet Glob Health 2015;3:S36.
33 Eberly LA, Rusangwa C, Ng'ang'a L, et al. Cost of integrated chronic care for severe non-communicable diseases at district hospitals in rural Rwanda. BMJ Glob Health 2019;4:e001449.

34 Jasem D, Majaliwa ES, Ramaiya K, et al. Incidence, prevalence and clinical manifestations at onset of juvenile diabetes in Tanzania. Diabetes Res Clin Pract 2019;156:10787

35 Marshall SL, Edidin D, Arena VC, et al. Prevalence and incidence of clinically recognized cases of Type 1 diabetes in children and adolescents in Rwanda, Africa. Diabet Med 2015;32:1186-92.

36 Pastakia SD, Karwa R, Kahn CB, et al. The evolution of diabetes care in the rural, resource-constrained setting of Western Kenya. Ann Pharmacother 2011;45:721-6.

37 Codner E, Acerini CL, Craig ME, et al. ISPAD clinical practice consensus guidelines 2018: what is new in diabetes care? Pediatr Diabetes 2018;19 Suppl 27:5-6.

38 American Diabetes Association. 9. Pharmacologic Approaches to Glycemic Treatment: Standards of Medical Care in Diabetes-2020. Diabetes Care 2020;43:S98-110.

39 Codner E, Acerini C, Craig ME, et al. ISPAD clinical practice consensus guidelines 2018: introduction to the limited care guidance appendix. Pediatr Diabetes 2018;19 Suppl 27:326-7.

40 Codner E, Acerini CL, Craig ME, et al. ISPAD clinical practice consensus guidelines 2018: limited care guidance appendix. Pediatr Diabetes 2018;19 Suppl 27:328-38.

41 Zargar $\mathrm{AH}$. Type 1 diabetes guidelines: are they enough? Indian $J$ Endocrinol Metab 2015;19:18-21. 